\title{
Controlling directed transport in two-dimensional periodic structures under crossed electric fields
}

\author{
S. Sengupta ${ }^{a}$, R. Guantes ${ }^{a}$, S. Miret-Artés ${ }^{a, *}$, P. Hänggi ${ }^{b}$ \\ anstituto de Matemáticas y Física Fundamental, Consejo Superior de Investigaciones Científicas, \\ Serrano, 123, 28006 Madrid, Spain \\ bInstitut für Physik, Universität Augsburg, Universitäts strasse 1, D-86135 Augsburg, Germany
}

Received 16 January 2004; received in revised form 6 February 2004

\begin{abstract}
We investigate different mechanisms for the control of directed transport of particles on twodimensional periodic and symmetric substrates, based on the application of a crossed static and a bi-harmonic (harmonic mixing) field. We focus on inertial systems in the low friction regime, using a prototypical model for atom-surface diffusion, and demonstrate that a proper control of current reversals can be achieved at moderate field strengths by tuning either the static field or changing the relative phase of the harmonic mixing signal, respectively.
\end{abstract}

(C) 2004 Elsevier B.V. All rights reserved.

PACS: 05.60. - k; 05.45.Ac; 05.45.Pq; 87.16.Nn; 32.80. - t

Keywords: Directed transport; Inertial ratchets in 2-D; Control of surface features; Chaotic transport; Current reversals; Brownian motors

\section{Introduction}

Transport phenomena in periodic potentials are ubiquitous in fields such as physics, chemistry and biology [1]. For systems wherein the noise intensity (for instance the scaled temperature $k T$ ) is significantly smaller than the transport barriers, the transport is governed by noise-assisted activation as described by rate theory in condensed media [2-4] with periodic potential landscapes.

Renewed interest in the field of directed transport stems from the theme of ratchet physics where unbiased, noise-induced transport emerges away from thermal

\footnotetext{
* Corresponding author. Tel.: +34-91-561-68-00 x 1101; fax: +34-91-585-48-94.

E-mail address: s.miret@imaff.cfmac.csic.es (S. Miret-Artés).
} 
equilibrium as a result of the action of Brownian motors [5-7]. Likewise, deterministic directed transport can result in time-dependent driven systems that exhibit a symmetry breaking of either spatial (ratchet potential) or of dynamic origin. This area has witnessed many interesting new developments and has found way into novel applications for directing, selecting or pumping particles on the mesoscopic and microscopic scale $[8,9]$. The technological applications cover mainly classical transport applications [8], but extend as well into the quantum domain $[8,10]$.

The vast majority of present theoretical and experimental ratchet studies have focused on one-dimensional geometries. In recent years, however, a variety of two-dimensional systems [11-16,18-20] and even three-dimensional microfluidic, intriguing Brownian motor systems have caught the attention of theoretical [21] and experimental [22] practitioners of ratchet physics. As has been pointed out in Ref. [16], two-dimensional systems possess the option to enhance and/or to control the symmetry breaking and the resultant transport features such as rectification. Ratchet physics has also found its way into the separation of biological molecules by use of time-varying fields through two-dimensional arrays of obstacles [23], while rectification features have been observed [16] for overdamped particles interacting with two-dimensional symmetric periodic substrates driven with a dc-drive along one direction and a circular ac-drive. In fact, a device has recently been fabricated that is able to control a priori the motion of magnetic flux quanta on a tailored niobium superconducting film [17].

In the present work we study an inertial system in the underdamped domain, with a two-dimensional symmetric potential driven by a constant bias field in combination with a time-periodic harmonic mixing field that acts along perpendicular directions. A harmonic mixing signal consists of two cosinusoidal periodic coherent perturbations at a fundamental frequency and its second harmonic. This perturbation is of vanishing mean but possesses nonvanishing, odd-numbered moments (dynamical symmetry breaking [24]). The overdamped limit with harmonic mixing signals in one-dimensional, spatially periodic potentials has been studied classically and quantum mechanically before [25]. Our present class of systems also generalizes the physics at work in dissipative one-dimensional, deterministic inertial ratchet systems exhibiting chaotic dynamics [26-29,31,32].

In a recent work [19] some of us investigated the diffusive and directed transport in this system under a single ac field, and observed intriguing current reversals. With this work, we extend our previous studies with our objective centering on the possibility to control at will the current in the two-dimensional plane, via the mutual interplay of harmonic mixing-induced directed transport and an externally applied bias force in the perpendicular direction. The tunable parameters that can readily be used for control are the frequency of the periodic signal, the relative strength of the two forcing amplitudes and its relative phase. This manipulation of signal parameters is in practice much simpler to implement as compared to a method where one tinkers with the ratchet potential parameters. The underlying potential is given by the experimental facts and often is symmetric by nature; see below. Thus the symmetry breaking must be induced dynamically by the time-dependent driving force.

The initial motivation to study this system emerges from the possibility to control the migration of atomic and molecular adsorbates on surfaces using electric fields (surface 
electro-migration [33]). First applications of the ratchet effect to electromigration are hinted at in the theoretical studies by Kehr [34] and more explicitly by Derényi et al. [35], who predicted a surface smoothing during growth when applying an ac-electric field. This phenomenon has been corroborated already experimentally [36]. The ratchet effect in those theoretical one-dimensional studies arises because of the spatial asymmetry of the potential at step edges (the Schwoebel barrier). One-dimensional studies of diffusion under weak electric fields have also been put forward by Talkner et al. [37]. Recent scanning tunnelling microscopy (STM) experiments have shown that electric fields can change the diffusion mechanisms [38] or induce rotations of molecules adsorbed on surfaces [39], opening the possibility to mechanically manipulate individual molecules.

In this case, we consider the transport of atoms on large terraces represented by a two-dimensional symmetric surface, and address the question of how to control the motion in either direction by applying crossed fields. The classical dynamics is described by the couple of equations (in scaled units) ${ }^{1}$

$$
\begin{gathered}
\ddot{x}=-\frac{\partial V(x, y)}{\partial x}-b \dot{x}-f_{x}(t), \\
\ddot{y}=-\frac{\partial V(x, y)}{\partial y}-b \dot{y}-f_{y},
\end{gathered}
$$

where the potential has the form [19]

$$
\begin{aligned}
V(x, y)= & \frac{1}{4 \pi^{2}}\left\{\left(V_{r}+1\right)-V_{r}[\cos (2 \pi x)+\cos (2 \pi y)]\right. \\
& \left.+\left(V_{r}-1\right) \cos (2 \pi x) \cos (2 \pi y)\right\} .
\end{aligned}
$$

The Wigner-Seitz cell for this potential has a minimum at $V(0,0)=0$ and a barrier for transport along the $x$ - or $y$-direction at $V\left( \pm \frac{1}{2}, 0\right)=V\left(0, \pm \frac{1}{2}\right)=\frac{1}{2} \pi^{2}$. The parameter $V_{r}$ is half the ratio between the maximum and the saddle-point of the nonscaled potential [19], and determines the barrier for transport along the diagonal directions, $V\left( \pm \frac{1}{2}, \pm \frac{1}{2}\right)=V_{r} / \pi^{2}$, and the strength of the potential coupling (for $V_{r}=1$ the potential is separable). The value of the friction coefficient $b$ and of the parameter $V_{r}$ are usually fixed by the experimental conditions. Here we set $b=0.07$ and $V_{r}=\frac{5}{8}$, which correspond to a model for diffusion of $\mathrm{Na}$ atoms on a symmetric $\mathrm{Cu}(001)$ surface fitted to experiment [40]. This very system operates in the low friction regime.

The ac-driver $f_{x}(t)$ producing the harmonic mixing signal is chosen to be of the form

$$
f_{x}(t)=e_{1} \cos (\omega t)+e_{2} \cos (2 \omega t+\phi)
$$

with $\omega=0.75$ fixed throughout this work, while $f_{y}$ is a static field. Note that there is no dc component in the $x$-direction, therefore rectification in $x$ is due to a ratchet effect. The amplitudes $f_{y}, e_{1}, e_{2}$ and the phase $\phi$ are the tunable parameters we use

\footnotetext{
${ }^{1}$ Distances are divided by the spatial period $a$ and times multiplied by the natural frequency of vibration $\omega_{0}=2 \pi \sqrt{V_{s} / 2 m a^{2}}$, where $m$ is the particle mass and $V_{s}$ the barrier for transport along the symmetric $x$ - and $y$-directions.
} 
to control the transport process. These are also easily varied in most experimental situations. In Sections 1 and 2 the phase is set equal to zero, and $e_{2}=0.05$ is fixed whenever we consider $f_{x}(t) \neq 0$. The role played by the phase is addressed in Section 3. The quantity of interest here is the net current of particles, defined by

$$
\langle\mathbf{v}\rangle \equiv \lim _{t \rightarrow \infty} \frac{\langle\mathbf{x}(t)-\mathbf{x}(0)\rangle}{t}=\lim _{n \rightarrow \infty} \frac{\langle\mathbf{x}(n T)-\mathbf{x}(0)\rangle}{n T},
$$

taking as unit of time the driver period $T=2 \pi / \omega$.

\section{Onset of directed transport under crossed fields}

Before investigating the control of transport in the 2D plane, it is interesting to discuss the onset of directed motion in this underdamped system due to both dc- and ac-fields, and the influence of the potential energy coupling. Consider first the separable $\left(V_{r}=1\right)$ case. Along $y$, where we have only a static field component, there are two thresholds for the onset of directed transport. The first one is given by the condition that the net energy change per spatial period in a limit cycle is enough to surmount the potential barrier [1], which for potential (2) is $f_{y}^{1}=2 b / \pi^{2} \sim 0.014$. The second threshold corresponds to the case where the effective potential $V_{\text {eff }}(0, y)=V(0, y) \pm f_{y} y$ has no minima, $f_{y}^{2}=1 / 2 \pi \sim 0.16$. For $f_{y} \geqslant f_{y}^{2}$, only running solutions of the equations of motion (1) are possible, while for $f_{y}^{1} \leqslant f_{y} \leqslant f_{y}^{2}$ we have hysteretic behavior between a pinned and a running solution [1]. The hysteresis region is marked with dashed lines in Fig. 1. We notice that for the corresponding overdamped system only the second threshold is present; consequently, the onset of directed transport takes place at much higher field strengths compared to the underdamped case.

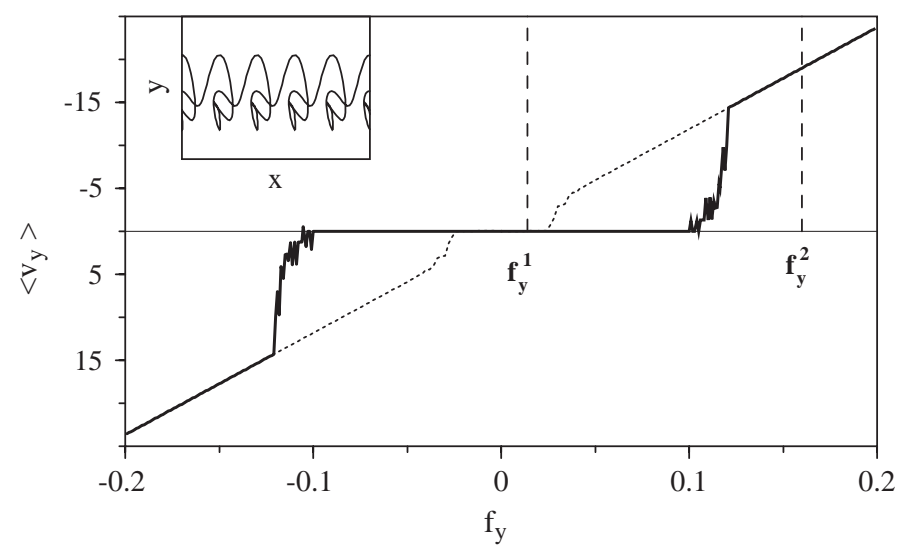

Fig. 1. Flux in the $y$-direction as a function of the static field $f_{y}$ for different amplitudes of the ac-field $f_{x}(t)$. Thick solid line: $e_{1}=e_{2}=0$. Dotted line: $e_{1}=0.083, e_{2}=0.05$. The threshold conditions $f_{y}^{1}=2 b / \pi^{2}$, $f_{y}^{2}=1 / 2 \pi$ for the separable potential are plotted with dashed lines (see text). The inset shows an attractor pinned in $y$ and running in $x$ for $f_{y}=0.03, e_{1}=0.083, e_{2}=0.05$. 
For the potential energy coupling considered here, $V_{r}=\frac{5}{8}$, by increasing the static field the pinned solution is more stable than the running one until $f_{y} \sim 0.12$ (Fig. 1, thick solid line). The direction of the flux in $y$ can be exactly inverted by changing the sign of $f_{y}$, due to the spatial symmetry. If we switch on the bi-harmonic signal in the perpendicular $x$-direction, it produces a significant effect on the net current along $y$, albeit there is no ac-component in this direction (Fig. 1, dotted line). Running solutions in $y$ are stabilized by the crossed field, and the onset of directed transport along $y$ takes place at $f_{y} \sim 0.02$, much closer to the $f_{y}^{1}$-threshold. Obviously, this is entirely due to the potential energy coupling. Moreover, we can observe pinned solutions in $y$; giving zero flux in this direction, and running in the perpendicular direction, as the one depicted in the inset of Fig. 1.

With respect to the $x$-direction, chaos does play a dominant role in the deterministic transport properties $[19,27-30]$. For $e_{2} \neq 0$ and any value of the phase $\phi$ one could in principle observe a ratchet effect in $x$, due to the breaking of the temporal symmetry $f_{x}(t+T / 2)=-f_{x}(t)$ [41]. Note, however, that in the underdamped limit $b \rightarrow 0$ one has to deal also with the symmetry $f_{x}(-t+T / 2)=f_{x}(t)$, and the condition, $\phi \neq 0$, $\pi$, may be required for inertial systems with very low friction values. Despite the fact that rectification is not ruled out, there can exist large intervals of the tunable amplitude $e_{1}$ where the net flux numerically is practically zero. This can be appreciated in Fig. 2, where we depict the current along $x$-direction and the stroboscopic bifurcation diagram in the corresponding velocity $v_{x}$. The existence of periodic or chaotic attractors for a given set of initial conditions is related to the presence-or not - of mode locking between the period of the particle inside the potential well and the period of the external driver [42].

The threshold conditions discussed above for the static field $f_{y}$ are only valid for the amplitude $e_{1}$ in the linear response approximation. However, they are important in establishing the existence of running orbits [19]. For instance, close to the value $e_{1}=f_{y}^{2} \sim 0.16$ running periodic attractors with period 1 appear. We remark that the mere existence of these attractors does not guarantee a net flux, since, for $e_{2}=0$, orbits traveling in opposite directions are equally stable and the average current is zero. For $e_{2} \neq 0$, and as a consequence of nonlinear response due to harmonic mixing (see Section 3), a particular orbit is more probable than others giving rise to net flux and current reversals. This is what we observe in Fig. 2a for $0.16 \leqslant e_{1} \leqslant 0.18$. An increase of the amplitude $e_{2}$ leads to stabilization of specific periodic attractors $[19,30]$. Here we seek different stabilization mechanisms, while trying to control the transport for moderate field strengths below the threshold $f_{y}^{2}$, a goal that is not possible within overdamped systems. This also is a more favorable situation in real experiments where strong field amplitudes are more difficult to achieve.

In Fig. 2b, we depict how the crossed static field affects the dynamics in $x$. It stabilizes wide chaotic regions, and can drastically change the transport features in the perpendicular direction $x$ by favoring different attractors, as can be seen in the top panel of this figure. In fact, most of the periodic attractors observed in the regular windows in Fig. 2a (where $f_{y}=0$ ) are no longer strictly periodic for $f_{y}=0.1$. They form narrow quasi-periodicity bands in the bifurcation diagram, due to the strong drift motion in $y$. In the next section we discuss how the static field plays a constructive 


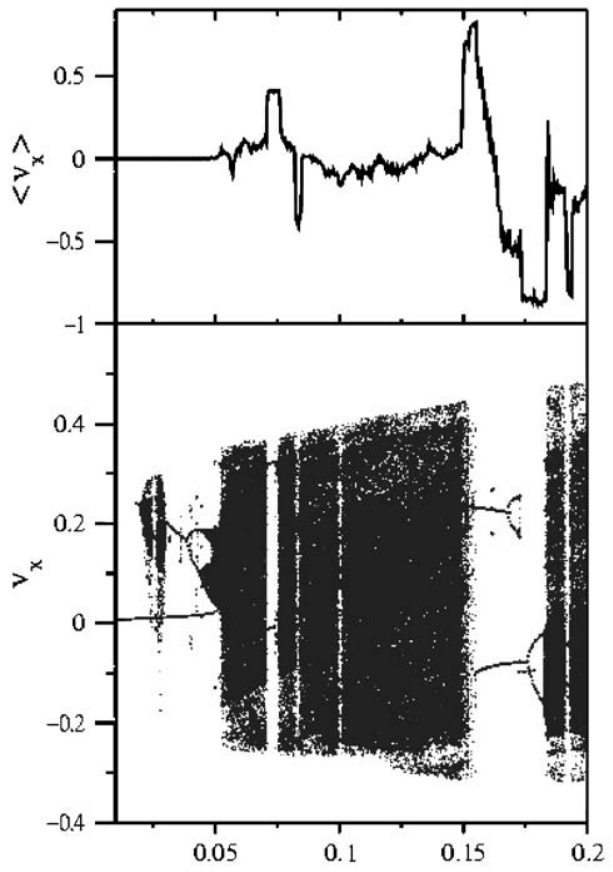

(a)

$\mathrm{e}_{1}$

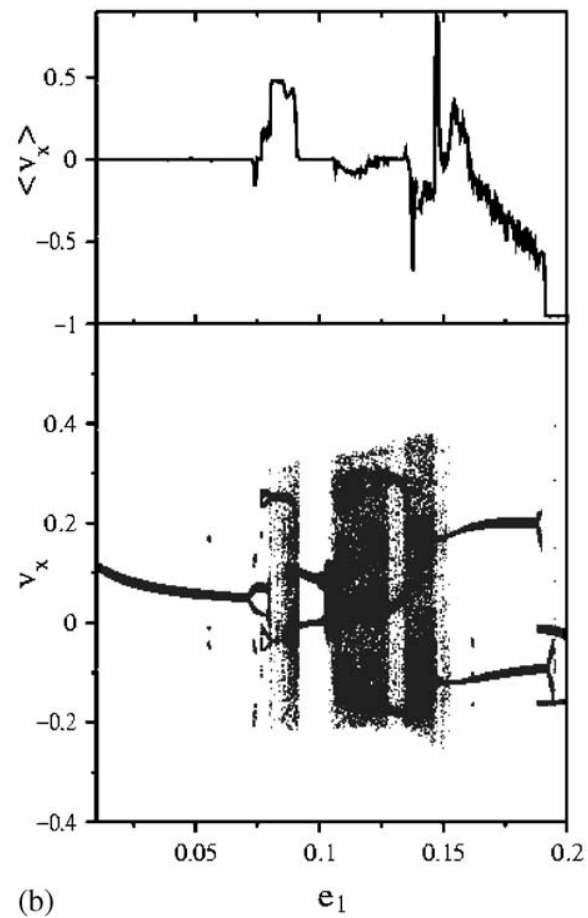

Fig. 2. Flux in the $x$-direction (top panels) and the corresponding bifurcation diagrams of the individual particle velocities as a function of the first harmonic amplitude $e_{1}$ of the harmonic mixing field, for different values of the static field applied along the $y$-direction: (a) $f_{y}=0$. (b) $f_{y}=0.1$.

role in order to control the directed motion induced by the harmonic mixing signal acting in $x$-direction.

\section{Control of directed transport generated by a crossed static field}

Here we choose some specific values of the ac-field amplitude $e_{1}$ shown in Fig. 2, and investigate in detail the effect induced by the static field $f_{y}$ in the transport properties. In Fig. 3 we plot again the current along the $x$-direction and the corresponding bifurcation diagram of $v_{x}$, this time as a function of $f_{y}$, for $e_{1}=0.083$ (a), and $e_{1}=0.095$ (b), respectively. In the first case, we note that a current reversal in $x$ occurs by tuning the field in $y$, from $\left\langle v_{x}\right\rangle=-\frac{1}{2}$ to $\left\langle v_{x}\right\rangle=\frac{1}{2}$. An inspection of the periodic solutions seen in the corresponding bifurcation diagram reveals the mechanism for this reversal: there are two competing running attractors of period 2, plotted in Fig. 4a, with opposite velocities. Upon inspecting Fig. 3 a the current reverses its sign around for $f_{y}=0.042$, where a bifurcation from a regular periodic orbit to a chaotic attractor occurs. The corresponding chaotic region comprises the interval $f_{y} \in[0.042,0.024]$ within which the current undergoes its sign change from $\frac{1}{2}$ towards $-\frac{1}{2}$. Due to 

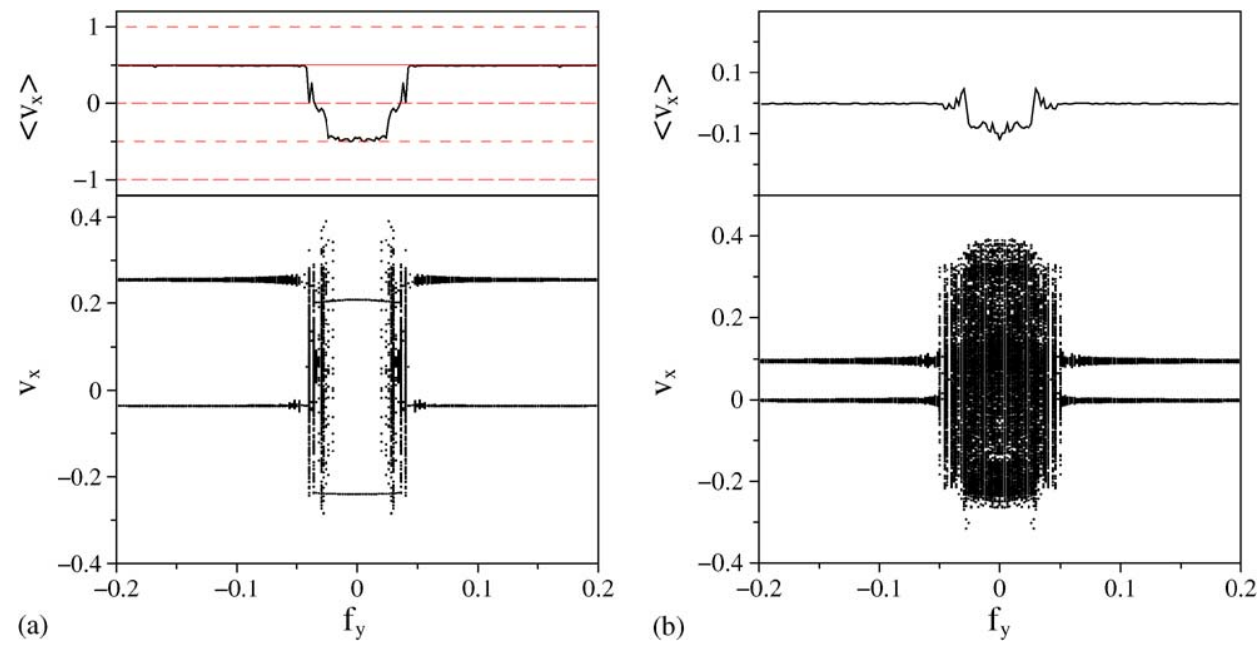

Fig. 3. Flux in $x$-direction and the corresponding bifurcation diagrams as a function of the static field $f_{y}$, for fixed amplitudes of the bi-harmonic field along $x$ : (a) $e_{1}=0.083, e_{2}=0.05$ and (b) $e_{1}=0.095, e_{2}=0.05$.
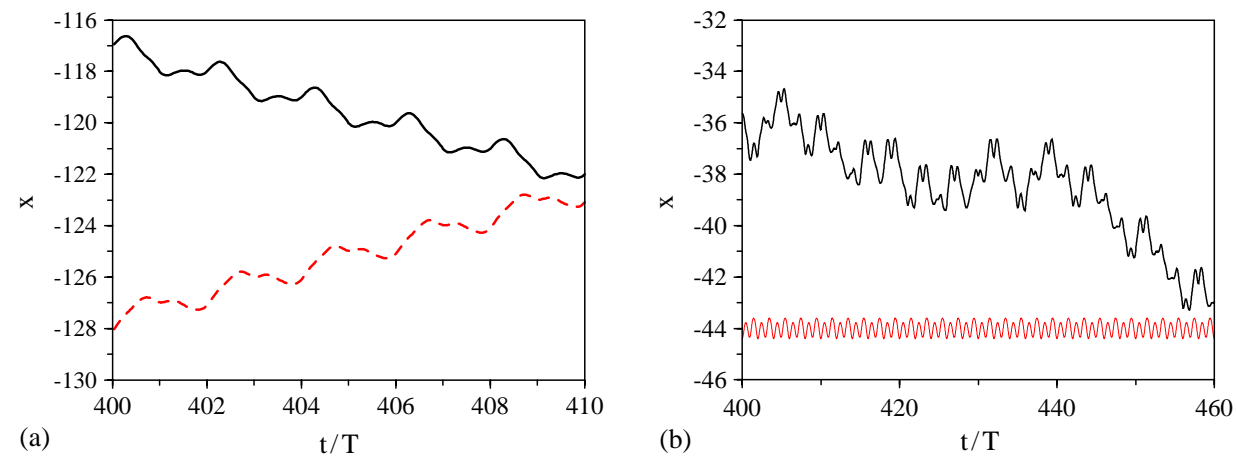

Fig. 4. Attractors corresponding to different ac- and dc-field strengths shown in Fig. 3. (a) $e_{1}=0.083, e_{2}=0.05$. Solid line: $f_{y}=0$. Dashed line: $f_{y}=0.1$ (see Fig. 3a). (b) $e_{1}=0.095, e_{2}=0.05$. Solid line: $f_{y}=0$. Dashed line: $f_{y}=0.1$ (see Fig. $3 b$ ).

symmetry, the same phenomenon occurs again for corresponding negative valued $f_{y}$; see in Fig. 3a. It should be emphasized that this mechanism is precisely the same that is at work in one-dimensional chaotic ratchets, as it has been investigated by Mateos in Refs. $[27,28]$.

Note that the inherent symmetry structure in the equations of motion in $(1,2)$ imply that the direction of the current in $x$ is independent of the sign of $f_{y}$ while the direction in $y$ is the opposite; compare also with Fig. 1. Next, we discuss a simple method to control the direction of the current in $x$ without changing the static field strength. 
In Fig. $3 \mathrm{~b}$, for a slightly larger value of the amplitude $e_{1}$, we have a completely different phenomenon: a region with average negative flux in $x$ yields no directed transport upon increasing (decreasing) the field $f_{y}$. This is again readily understood upon inspecting the corresponding attractors, see Fig. 4b. For zero static field, we have running chaotic solutions with a small bias in the negative $x$ direction. A nonzero field $f_{y}$ stabilizes the chaotic motion in $x$, rendering it periodic, but pinned in this direction, yielding no net current. Notice that this solution is the opposite case to that depicted in the inset of Fig. 1, where a particle is pinned in $y$ while running in $x$. Therefore, by tuning properly the crossed static field $f_{y}$ we can completely control the transport along the $x$-direction.

\section{Phase control of directed transport}

For a one-dimensional ratchet potential with a bi-harmonic ac-field, it has been shown by Barbi et al. [30] that the relative phase of the field affects considerably the directed transport. This is due to the fact that there is an interplay between the dynamical symmetry breaking due to harmonic mixing and the ratchet effect induced by the asymmetric potential landscape. Here, all the rectification along $x$ is entirely due to the time-dependent signal, and therefore the phase can be used to change the transport in a controllable way.

The existence of a net current in $x$ can be predicted by calculating the response of the system to the external field, which is proportional to the average velocity $[1,43]$. One can expand the adiabatic response function $R$ in a Taylor series in $f_{x}(t)$,

$$
R\left(f_{x}\right)=R_{1} f_{x}+R_{2} f_{x}^{2}+R_{3} f_{x}^{3}+\cdots .
$$

For $e_{2} \neq 0$, the leading nonzero contribution to the average response is given by the third-order term

$$
\bar{R}=\frac{3}{2} \pi R_{3} e_{1}^{2} e_{2} \cos \phi+\cdots,
$$

Then, we deduce that a response equal in magnitude but opposite in sign is obtained by a phase shift $\phi \rightarrow \phi+\pi$. This is verified numerically in Fig. 5 calculating the net flux in $x$ as a function of $\phi$. We use the same parameter set for the bi-harmonic field than used for Fig. $3 \mathrm{a}, e_{1}=0.083, e_{2}=0.05$, and different static field strengths. Thus, one can readily reverse the direction of the motion by imposing a relative phase change.

\section{Conclusions}

In the present work, we have explored the control possibilities of crossed electric fields on the directed transport of particles moving on two-dimensional periodic substrates. To this end we have focused on an underdamped system in the low friction, moderate field regime (for this system, a field strength $f \sim 0.05$ produces an energy gain equal to the barrier for transport), generalizing previous studies on $2 \mathrm{D}$ overdamped 


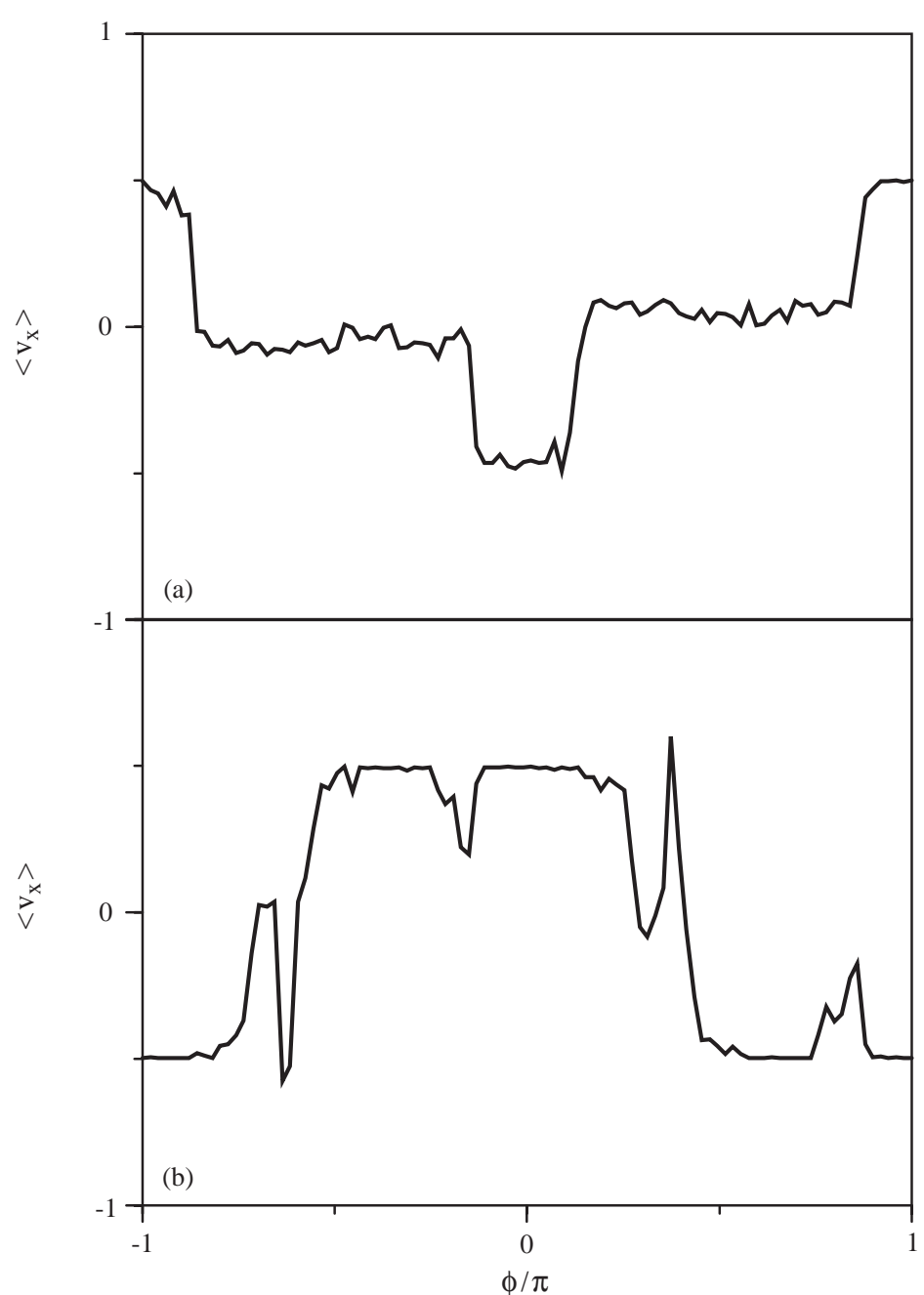

Fig. 5. Flux in the $x$-direction as a function of the relative phase $\phi$ of the bi-harmonic signal, for $e_{1}=0.083$, $e_{2}=0.05$ (see Fig. 3a). (a) $f_{y}=0$ and (b) $f_{y}=0.1$.

systems [16] with different crossed fields. We have also chosen a static field in one direction and a harmonic mixing signal in the other one, emphasizing the role of the potential energy coupling in the way each field affects the transport in the perpendicular direction. The system investigated represents a model for transport of $\mathrm{Na}$ atoms on a $\mathrm{Cu}(001)$ surface [40], therefore the results presented here can be of relevance for the control of surface electromigration [33,35,38], but are also applicable to particle separation in $2 \mathrm{D}$ devices $[15,21,23]$, transport of vortices in superconductors $[17,44]$ or electron currents through semiconductors [9]. 
We have demonstrated that by using as tuning parameters the static field strength $f_{y}$, or the first harmonic amplitude $e_{1}$, as well as the relative phase of the ac-field, a detailed control of the directed transport in the 2D-plane is possible. The realization of this control can likely be more simply implemented in experiments than a method which is based on re-structuring the symmetry of the surface potential. The symmetry breaking brought about by the second harmonic signal leads to rectification effects while the crossed steady field provides an appealing flexibility in controlling this transport. In particular, current reversals in the $x$-direction can be obtained by changing the static field in $y$, due to stabilization of different attractors. A crossed field can also pin a running solution in the perpendicular direction causing the resultant current to vanish. Because of the nonlinear, phase-dependent response to the bi-harmonic signal the relative phase can be used to change a priori the direction of the current in $x$-direction.

The approach pursued here also generalizes some recent proposals for control and manipulation of 1D Hamiltonian and weakly damped inertial systems [45,46] by symmetry breaking.

\section{Acknowledgements}

This work has been supported in part (S.M.-A.) by DGICYT (Spain) under contract BFM2001-2179, and in part (P.H.) by the Deutsche Forschungsgemeinschaft, Grant HA-1517/13-4. R.G. thanks the Ministry of Science and Technology (Spain) for a Ramón y Cajal contract. S.S. acknowledges the Ministry of Education, Culture and Sports (Spain) for its 'Mobility of Foreign Researchers' program, Grant SB2001-0196.

\section{References}

[1] H. Risken, The Fokker-Planck Equation, Springer, Berlin, 1989.

[2] P. Hänggi, F. Mojtabai, Phys. Rev. A 26 (1982) 1168;

P. Hänggi, J. Stat. Phys. 42 (1986) 105;

P. Hänggi, J. Stat. Phys. 44 (1986) 1003.

[3] P. Hänggi, P. Talkner, M. Borkovec, Rev. Mod. Phys. 62 (1990) 251.

[4] V.I. Melnikov, Phys. Rep. 209 (1991) 1.

[5] P. Hänggi, R. Bartussek, Lecture Notes Phys. 476 (1996) 294.

[6] R.D. Astumian, P. Hänggi, Phys. Tod. 55 (11) (2002) 33.

[7] P. Reimann, P. Hänggi, Appl. Phys. A 75 (2002) 169.

[8] P. Reimann, Phys. Rep. 361 (2002) 57.

[9] H. Linke, Appl. Phys. A 75 (2002) 167 (Special Issue: Ratchets and Brownian Motors: Basics, Experiments and Applications).

[10] P. Reimann, M. Grifoni, P. Hänggi, Phys. Rev. Lett. 79 (1997) 10.

[11] A.W. Ghosh, S.V. Khare, Phys. Rev. Lett. 84 (2000) 5243.

[12] J.-D. Bao, Y.Z. Zhuo, Phys. Lett. A 239 (1998) 228; H.-Y. Wang, J.-D. Bao, Physica A 323 (2003) 197.

[13] A. Igarashi, H. Goko, S. Tsukamoto, Physica A 325 (2003) 62.

[14] U. Erdmann, W. Ebeling, V.S. Anishchenko, Phys. Rev. E 65 (2002) 061106.

[15] M. Kostur, L. Schimansky-Geier, Phys. Lett. A 265 (2000) 337;

M. Bier, M. Kostur, I. Derényi, R.D. Astumian, Phys. Rev. E 61 (2000) 7184;

I. Derényi, R.D. Astumian, Phys. Rev. E 58 (1998) 7781. 
[16] C. Reichhardt, C.J. Olson, M.B. Hastings, Phys. Rev. Lett. 89 (2002) 024101;

C. Reichhardt, C.J.O. Reichhardt, Phys. Rev. E 68 (2003) 046102.

[17] J.E. Villegas, S. Savel'ev, F. Nori, E.M. Gonzales, J.V. Anguita, R. Garcia, J.L. Vincent, Science 302 (2003) 1188.

[18] R. Eichhorn, P. Reimann, P. Hänggi, Phys. Rev. Lett. 88 (2002) 190601;

R. Eichhorn, P. Reimann, P. Hänggi, Phys. Rev. E 66 (2002) 066132;

R. Eichhorn, P. Reimann, P. Hänggi, Physica A 325 (2003) 101.

[19] R. Guantes, S. Miret-Artés, Phys. Rev. E 67 (2003) 046212.

[20] A. Sterck, S. Weiss, D. Koelle, Appl. Phys. A 75 (2002) 253.

[21] C. Kettner, P. Reimann, P. Hänggi, F. Müller, Phys. Rev. E 61 (2000) 312.

[22] S. Matthias, F. Müller, Nature 424 (2003) 53.

[23] D. Ertas, Phys. Rev. Lett. 80 (1998) 1548;

T.A.J. Duke, R.H. Austin, Phys. Rev. Lett. 80 (1998) 1552.

[24] J. Luczka, R. Bartussek, P. Hänggi, Europhys. Lett. 31 (1995) 431;

P. Hänggi, R. Bartussek, P. Talkner, J. Luczka, Europhys. Lett. 35 (1996) 315.

[25] W. Wonneberger, Solid State Commun. 30 (1979) 511;

F. Marchesoni, Phys. Lett. A 119 (1986) 221;

I. Goychuk, P. Hänggi, Europhys. Lett. 43 (1998) 503.

[26] P. Jung, J.G. Kissner, P. Hänggi, Phys. Rev. Lett. 76 (1996) 3436.

[27] J.L. Mateos, Phys. Rev. Lett. 84 (2000) 258.

[28] J.L. Mateos, Physica D 168 (2002) 205.

[29] M. Barbi, M. Salerno, Phys. Rev. E 62 (2000) 1988

[30] M. Barbi, M. Salerno, Phys. Rev. E 63 (2001) 066212.

[31] H.A. Larrondo, C.M. Arizmendi, F. Family, Physica A 320 (2003) 119;

C.M. Arizmendi, F. Family, A.L. Salas-Brito, Phys. Rev. E 63 (2001) 061104.

[32] J.L. Mateos, Physica A 325 (2003) 92.

[33] P.J. Rous, T.L. Einstein, E.D. Williams, Surf. Sci. 315 (1994) L995;

H. Yasunaga, A. Natori, Surf. Sci. Rep. 15 (1992) 205.

[34] K.W. Kehr, K. Mussawisade, T. Wichmann, W. Dietrich, Phys. Rev. E 56 (1997) R2351.

[35] I. Derényi, C. Lee, A.L. Barabási, Phys. Rev. Lett. 80 (1998) 1473;

I. Derényi, Appl. Phys. A 75 (2002) 217.

[36] P.J. de Pablo, J. Colchero, J. Gómez-Herrero, A. Asenjo, M. Luna, P.A. Serena, A.M. Baró, Surf. Sci. $464(2000) 123$

P.J. de Pablo, A. Asenjo, J. Colchero, P.A. Serena, J. Gómez-Herrero, A.M. Baró, Surf. Interface Anal. $30(2000) 278$.

[37] P. Talkner, E. Hershkovitz, E. Pollak, P. Hänggi, Surf. Sci. 437 (1999) 198;

M. Borromeo, F. Marchesoni, Surf. Sci. 465 (2000) L771.

[38] L.M. Sanders, R. Stumpf, T.R. Mattson, B.S. Swartzentruber, Phys. Rev. Lett. 91 (2003) 206104; J.M. Carpinelli, B.S. Swartzentruber, Phys. Rev. B 58 (1998) R13423.

[39] J.K. Gimzewski, C. Joachim, Science 283 (1999) 1683.

[40] J. Ellis, A.P. Graham, F. Hofmann, J.P. Toennies, Phys. Rev. B 63 (2001) 195408.

[41] S. Flach, O. Yevtushenko, Y. Zolotaryuk, Phys. Rev. Lett. 84 (2000) 2358;

P. Reimann, Phys. Rev. Lett. 86 (2001) 4992.

[42] D. D'Humieres, M.R. Beasley, B.A. Huberman, A. Libchaber, Phys. Rev. A 26 (1982) 3483.

[43] S. Denisov, S. Flach, A.A. Ovchinnikov, O. Yevtushenko, Y. Zolotaryuk, Phys. Rev. E 66 (2002) 041104.

[44] C. Reichhardt, C.J. Olson, M.B. Hastings, Phys. Rev. Lett. 89 (2002) 024101;

J.F. Wambaugh, C. Reichhardt, C.J. Olson, F. Marchesoni, F. Nori, Phys. Rev. Lett. 83 (1999) 5106.

[45] S. Denisov, J. Klafter, M. Urbakh, Phys. Rev. E 66 (2002) 046203.

[46] H.A. Larrondo, F. Family, C.M. Arizmendi, Physica A 303 (2002) 67. 